\title{
Roles of nuclear weak rates on the evolution of degenerate cores in stars
}

\author{
Toshio Suzuki ${ }^{1,2, \star}$, Naofumi Tsunoda ${ }^{3}$, Yuhsuke Tsunoda ${ }^{3}$, Noritaka Shimizu ${ }^{3}$, and Takaharu \\ Otsuka ${ }^{4}$ \\ ${ }^{1}$ Department of Physics, College of Humanities and Sciences, Nihon University, Sakurajosui 3-25-40, \\ Setagaya-ku, Tokyo 156-8550, Japan \\ ${ }^{2}$ National Astronomical Observatory of Japan, Osawa 2-21-1, Mitaka, Tokyo 181-8588, Japan \\ ${ }^{3}$ Center for Nuclear Study, The University of Tokyo, Hongo, Tokyo 113-0033 and Wako, Saitama 351-0198, \\ Japan \\ ${ }^{4}$ RIKEN Nishina Center for Accelerated-Based Science, Hirosawa 2-1, Wako, Saitama 351-0198, Japan
}

\begin{abstract}
Electron-capture and $\beta$-decay rates in stellar environments are evaluated with the use of new shell-model Hamiltonians for $s d$-shell and $p f$-shell nuclei as well as for nuclei belonging to the island of inversion. Important role of the nuclear weak rates on the final evolution of stellar degenerate cores is presented. The weak interaction rates for $s d$-shell nuclei are calculated to study nuclear Urca processes in $\mathrm{O}-\mathrm{Ne}-\mathrm{Mg}$ cores of stars with 8-10 $\mathrm{M}_{\odot}$ (solar mass) and their effects on the final fate of the stars. Nucleosynthesis of iron-group elements in Type Ia supernova explosions are studied with the weak rates for $p f$-shell nuclei. The problem of the neutron-rich iron-group isotope over-production compared to the solar abundances is shown to be nearly solved with the use of the new rates and explosion model of slow defraglation with delayed detonation. Evaluation of the weak rates is extended to the island of inversion and the region of neutron-rich nuclei near ${ }^{78} \mathrm{Ni}$, where two major shells contribute to their configurations.
\end{abstract}

\section{Introduction}

Roles of the rate of nuclear processes driven by electron-capture and $\beta$-decays on the final evolution of electron-degenerate cores of stars are investigated. Standard electron-capture and $\beta$-decay rates of nuclei presented in a recent literature [1] are those for (1) $s d$-shell nuclei by [2], (2) $p f$-shell of LMP [3], (3) $p f-g$-shell of LMSH [4], and (4) Fuller-Fowler-Newman (FFN) rates [5]. Besides them, an approximate two-parameter formula for the rates is obtained [6] to be used for nuclei, which have no available data tables.

Here, we study nuclear Urca processes in stars with $\mathrm{O}-\mathrm{Ne}-\mathrm{Mg}$ cores by employing a set of new weak rates computed by the USDB [7] for $s d$-shell nuclei. Nucleosynthesis of iron-group nuclei in Type Ia supernova explosions ( $\mathrm{SNe}$ ) (see [8] for a recent review on nucleosynthesis in Type Ia SNe and hydrodynamical explosion models) is discussed by using a new Hamiltonian GXPF1J [9] for $p f$ shell nuclei. We also extend our study to the weak rates for nuclei in the island of inversion, where

^e-mail: suzuki@phys.chs.nihon-u.ac.jp 
$s d$-shell and $p f$-shell components are considerably admixed, as well as those for $p f-g$-shell nuclei near ${ }^{78} \mathrm{Ni}$, which play important roles on nucleosynthesis in core-collapse $\mathrm{SNe}$.

\section{Weak rates in $s d$-shell and nuclear Urca processes in O-Ne-Mg cores}

Stars with 8-10 $\mathrm{M}_{\odot}$, where O-Ne-Mg cores form after carbon burning, may evolve in various ways such as O-Ne-Mg white dwarfs (WD), e-capture (EC) SNe or core-collapse (CC) SNe. The stellar fate is sensitive to their masses and nuclear e-capture and $\beta$-decay rates. Cooling of the $\mathrm{O}-\mathrm{Ne}-\mathrm{Mg}$ core by nuclear Urca processes determines whether the star ends up with ECSNe or CCSNe [10, 11].

Nuclear Urca processes for pairs of $s d$-shell nuclei, ${ }^{23} \mathrm{Ne}-{ }^{23} \mathrm{Na}$ and ${ }^{25} \mathrm{Na}-{ }^{25} \mathrm{Mg}$, are found to be important for cooling the O-Ne-Mg core $[10,11]$. Electron-capture and $\beta$-decay occur simultaneously at a certain density, called "Urca density", and stellar energy is lost by emissions of both $v$ and $\bar{v}$. At high densities electron chemical potential increases and e-capture on nuclei is ignited when it reaches the Q-value of the reaction. The Urca density determined by the Q-value of the reaction is found to be $\log _{10} \rho Y_{e}=8.92$ and 8.78 for ${ }^{23} \mathrm{Ne}^{23} \mathrm{Na}$ and ${ }^{25} \mathrm{Na}-{ }^{25} \mathrm{Mg}$ pairs, respectively.

Double e-capture reactions on ${ }^{24} \mathrm{Mg}$ or ${ }^{20} \mathrm{Ne}$, which are succeeded by e-capture on ${ }^{24} \mathrm{Na}$ or ${ }^{20} \mathrm{~F}$, respectively, are important for heating the core in later stages of the stellar evolution $[12,13]$. Electroncapture, $\beta$-decay and $v$ energy loss rates as well as energy production rates by $\gamma$ emissions are tabulated for $s d$-shell nuclei using a fine grid for density and temperature in [13].

\section{Electron-capture rates in $p f$-shell nuclei and nucleosynthesis in Type la $\mathrm{SNe}$}

New shell-model Hamiltonians in $p f$-shell, GXPF1 [14] and GXPF1J [9], can describe spin properties of $p f$-shell nuclei quite well. The Gamow-Teller (GT) strengths in Ni isotopes and M1 strengths in various $p f$-shell nuclei are also well described [15]. In particular, for ${ }^{56} \mathrm{Ni}$, the GT strength distribution is nicely reproduced $[15,16]$. In Type-Ia supernova explosions, a plenty amount of ${ }^{56} \mathrm{Ni}$ is produced. Production yields of neutron-rich nuclei and the lepton-to-baryon ratio (or proton fraction) $Y_{e}$ depend sensitively on the rate of e-capture on ${ }^{56} \mathrm{Ni}$. The ratio of the production yields of ${ }^{58} \mathrm{Ni}$ over ${ }^{56} \mathrm{Ni}$ can be reduced nearly by half for GXPF1J compared to KB3G [17].

The problem of over-production of ${ }^{58} \mathrm{Ni},{ }^{54} \mathrm{Cr}$ and ${ }^{54} \mathrm{Fe}$ compared to the solar abundance was discussed [18] with the use of the capture rates of FFN [5]. A possible solution of the problem with slower e-capture rates was discussed in $[3,19]$. The problem could be solved by using slower e-capture rates of GXPF1J. Here, we use the W7 and WDD2 model [18] as explosion models of Type-Ia supernova explosions starting from $\mathrm{C}-\mathrm{O}$ white dwarf with a mass $1.38 \mathrm{M}_{\odot}$. The $\mathrm{W} 7$ model proceeds by a fast deflagration while the WDD2 model proceeds by a slow deflagration with delayed detonation. Electron-capture rates of GXPF1J are used for $p f$-shell nuclei with $21 \leq Z \leq 32$ and those of KBF [3] otherwise. At high densities above a few $10^{8} \mathrm{gcm}^{-3}$, slower e-capture processes on Ni and $\mathrm{Fe}$ isotopes lead to less production of neutron-rich nuclei (see [20] for the details). The results of final element abundances are shown in Ref. [20]. In the WDD2 (W7) model, over-production of neutron-rich $\mathrm{Cr}, \mathrm{Fe}$ and $\mathrm{Ni}$ isotopes compared with the solar system abundances is suppressed within a factor of 2 (2-3). This is much smaller than the case of FFN weak rates, where the over-production factor becomes as large as up to 4-5.

\section{Weak rates for nuclei in the island of inversion}

Urca processes for nuclear pairs in the island of inversion [21] such as ${ }^{31} \mathrm{Mg}-{ }^{31} \mathrm{Al}$ and ${ }^{33} \mathrm{Mg}-{ }^{33} \mathrm{Al}$ pairs have been pointed out to be important for the cooling of neutron star crusts [22]. Large $s d-p f$ shell 
admixtures are found in neutron-rich $\mathrm{Ne}, \mathrm{Na}$ and $\mathrm{Mg}$ isotopes near $N=20$. Lowering of $2_{1}^{+}$states and enhancement of $E 2$ transition strengths show evidence for the breaking of the magicity at $N=20$ [21]. Energy levels of $2_{1}^{+}$states and $B\left(E 2: 2_{1}^{+} \rightarrow 0_{\text {g.s. }}^{+}\right)$are sucessfully reproduced by SDPF-M Hamiltonian [23] with $s d-p f$-shell configurations. The important contributions from $2 \mathrm{p}-2 \mathrm{~h}$ components are found in ${ }^{30} \mathrm{Ne}$ and ${ }^{32} \mathrm{Mg}$.
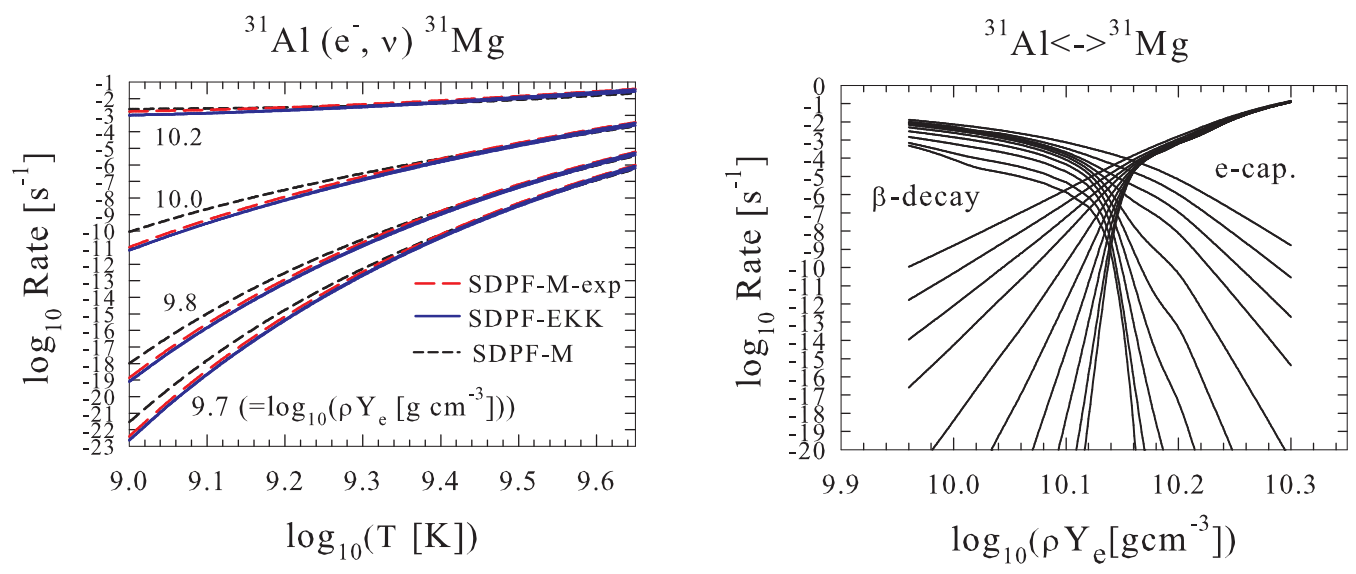

Figure 1. (Left) Electron-capture rates for ${ }^{31} \mathrm{Al}\left(\mathrm{e}^{-}, v\right){ }^{31} \mathrm{Mg}$ obtained with SDPF-M Hamiltonian (SDPF-M) and EKK method (SDPF-EKK). The rates obtained with SDPF-M including experimental data are denoted by SDPF-M-exp. (Right) Electron-capture and $\beta$-decay rates for ${ }^{31} \mathrm{Al}-{ }^{31} \mathrm{Mg}$ pair at various temperatures obtained by the EKK method.

We discuss the weak rates for ${ }^{31} \mathrm{Mg}-{ }^{31} \mathrm{Al}$ pair. The SDPF-M fails to reproduce the energy levels of ${ }^{31} \mathrm{Mg}$, that is, $7 / 2^{-}$state becomes the ground state while the experimental g.s. is $1 / 2^{+}$. The Urca density can not be clearly assigned for the weak rates for SDPF-M. This shortcoming can be improved for the effective interaction obtained by EKK (extended Kuo-Krenciglowa) method [24], which can properly treat Q-box calculations in two-major shells without divergence problems [25]. Energy levels in ${ }^{31} \mathrm{Mg}$ are well reproduced, and more important roles of $\mathrm{p}$-h excitations are noticed compared with the SDPF-M case. The $4 \mathrm{p}-4 \mathrm{~h}$ components are found to be as much as the $2 \mathrm{p}-2 \mathrm{~h}$ components in ${ }^{32} \mathrm{Mg}$. The weak rates are evaluated with the EKK method and they prove to be close to those obtained by taking into account the available experimental data as shown in Fig. 1. For the EKK approach, the Urca density can be assigned to be $\log _{10} \rho Y_{e}=10.14$.

\section{Summary}

New weak rates for $s d$-shell and $p f$-shell nuclei are obtained with USDB and GXPF1J Hamiltonians, respectively. The rates for $s d$-shell are used to study evolutions of the O-Ne-Mg cores of stars with 8-10 $\mathrm{M}_{\odot}$. Nuclear Urca processes for $A=23$ and 25 nuclear pairs are found to be important for the cooling of the cores and determination of the fate of the stars whether they end up with ECSNe or CCSNe. The rates for $p f$-shell are used to study nucleosynthesis of iron-group elements in Type Ia $\mathrm{SNe}$. Relatively smaller e-capture rates for GXPF1J compared with KB3G, KBF and FFN are found to considerably suppress the over-production of neutron-rich iron-group isotopes, especially in the WDD2 explosion model with detonation. The weak rates for GXPF1J are tabulated for further study of astrophysical processes [26]. 
Evaluation of new weak rates is extended to nuclei in the island of inversion, where substantial admixtures of both $s d$-shell and $p f$-shell components are noticed. Nuclear weak rates for ${ }^{31} \mathrm{Mg}-{ }^{31} \mathrm{Al}$ pair, important for Urca processes in neutron star crusts, are evaluated with a new interaction obtained by the EKK method. Study of nuclear structure and weak rates for $p f-g$-shell nuclei near ${ }^{78} \mathrm{Ni}$, which play important roles on nucleosynthesis in CCSNe, is in progress. RPA calculations give ecapture rates close to the rates of the approximate formula in Ref. [1]. Shell-model calculations within $p f-g_{9 / 2} d_{5 / 2}$-shell configurations can explain the excitation energy of the first $2^{+}$state in ${ }^{78} \mathrm{Ni}$ [27], but the configuration space needs to be extended to include the full $g d s$-shells to obtain reliable weak rates as dominant contributions come from spin-dipole forbidden transitions.

Nuclear weak rates, thus, play important role on the final evolution of degenerate cores in stars. Accurate evaluation of the weak rates is essential for the studies of various astrophysical processes sensitive to the rates.

The author would like to thank K. Nomoto, H. Toki, S. Jones, R. Hirschi, M. Honma, K. Mori, M. Famiano and T. Kajino for collaborations of the works in Sects. 2 and 3. This work has been supported in part by Grants-in-Aid for Scientific Research (C) 15K05090 of the MEXT of Japan.

\section{References}

[1] C. Sullivan et al., Astrophys. J. 816, 44 (2016).

[2] T. Oda et al., At. and Nucl. Data Tables 56, 231 (2002).

[3] K. Langanke and G. Martinez-Pinedo, At. And Nucl. Data Tables, 79, 1 (2001).

[4] K. Langanke, E. Kolbe and D. J. Dean, Phys. Rev. C 63, 032801 (2001).

[5] G. M. Fuller, W. Fowler and M. Newman, Astrophys. J. 252, 715 (1982).

[6] K. Langanke, G. Martinez-Pinedo, 2003, Rev. Mod. Phys. 75, 819 (2003).

[7] B. A. Brown and W. Richter, Phys. Rev. C 74, 034315 (2006).

[8] I. R. Seitenzahl and D. M. Townsley, Handbook of Supernovae (Springer, 2017).

[9] M. Honma et al., J. Phys. Conf. Ser., 20, 7 (2005).

[10] S. Jones et al., Astrophys. J. 772, 150 (2013).

[11] H. Toki, T. Suzuki, K. Nomoto, S. Jones, R. Hirsci, Phys. Rev. C 88, 015806 (2013).

[12] G. Martinex-Pinedo et al., Phys. Rev. C 89, 045806 (2014).

[13] T. Suzuki, H. Toki and K. Nomoto, Astrophys. J. 817, 163 (2016).

[14] M. Honma et al., Phys. Rev. C 69, 034335 (2004).

[15] T. Suzuki et al., Phys. Rev. C 83, 044619 (2011).

[16] M. Sasano et al., Phys. Rev. Lett. 107, 202501 (2011).

[17] J. Poves et al., Nucl. Phys. A 694, 157 (2001).

[18] K. Iwamoto et al., Astrophys. J. 125, 439 (1999).

[19] F. Brachwitz et al., Astrophys. J. 536, 934 (2001).

[20] K. Mori et al., Astrophys. J. 833, 179 (2016).

[21] E. K. Warburton, J. A. Becker and B. A. Brown, Phys. Rev. C 41, 1147 (1990).

[22] H. Schatz et al., Nature 505, 65 (2014).

[23] Y. Utsuno et al., Phys. Rev. C 60, 054315 (1999).

[24] N. Tsunoda et al., Phys. Rev. C 95, 021304(R) (2017).

[25] N. Tsunoda, K. Takayanagi, Hjorth-Jensen and T. Otsuka, Phys. Rev. C 89, 024313 (2014).

[26] M. Honma and T. Suzuki, http://www.phys.chs.nihon-u.ac.jp/suzuki/data4/link.html (2017).

[27] Y. Tsunoda et al., Phys. Rev. C 89, 031301(R) (2014). 